\title{
Intermediate Structures in Fission and Consequences on Average Partial Cross Sections
}

\author{
Olivier H. Bouland ${ }^{1, a}$ \\ ${ }^{1}$ CEA, DEN, DER, SPRC, Physics Studies Laboratory Cadarache, F-13108 Saint-paul- \\ lez-Durance, France
}

\begin{abstract}
This paper is willing to illustrate and quantify the impact of a double fission barrier in terms of average fission cross section and in particular, to status on both the additional class-II width correction factor and the degree of freedom of the overall double fission barrier which is a key parameter in the calculation of the standard $W_{n, f}$ factor of Hauser-Feshbach theory.
\end{abstract}

\section{Problematic related to average fission cross section simulation}

The purpose of this communication is to both recall and emphasize the existence of the class-II state width fluctuation factor, $W_{I I}$, whose treatment is usually disregarded in standard average cross section evaluation codes. The impact of fission channel Intermediate Structures (IS) is well pictured by Fig. 1 which displayed the observed and current evaluated fission cross sections within the neutron-incident Unresolved Resonance energy Range (URR) for a fissile and a fertile isotope borrowed from the plutonium family. Below the fission threshold energy $\left(E_{n}<500 \mathrm{keV}\right)$, we observe large manifestations of the IS (Fig. 1- right side), relatively to the fission cross section magnitude, although IS effects show up as well for fissile isotopes (Fig. 1- left side). We guess that more complexity in the classically [1] used URR fission cross section model will be required for a fertile isotope but are we allowed to make some approximations for a fissile isotope without collateral damage? This is the question raised by this paper. In the next sections, the impact of both the barrier tunneling and $W_{I I}$ factor for fissile and fertile isotopes is clarified using a new way of calculating the inextricably admixed IS and $W_{I I}$ effects based on accurate formal R-matrix Monte-Carlo-fashion calculations which have been recently applied to the whole plutonium family in a consistent and macro-microscopic approach [2].

\section{Standard Hauser-Feshbach formalism}

Reaction cross-sections in the URR are commonly calculated using standard Hauser-Feshbach theory [7]. The average fission cross section $\bar{\sigma}_{c f}$ for the entrance channel $c$ at neutron energy $E_{n}$ reads

$$
\bar{\sigma}_{c f}^{J^{\pi}}\left(E_{n}\right)=\sum_{J} \sigma_{c}^{J^{\pi}}\left(E_{n}\right) \times \bar{P}_{f} \times W_{c f}=\sum_{J} \sigma_{c}^{J^{\pi}}\left(E_{n}\right) \sum_{s^{\prime}=\left|I^{\prime}-i^{\prime}\right|}^{\left|I^{\prime}+i^{\prime}\right|} \sum_{l^{\prime}=\left|J-s^{\prime}\right|}^{\left|J+s^{\prime}\right|} \frac{T_{f}^{J^{\pi}\left(l^{\prime} s^{\prime}\right)}\left(E_{c^{\prime}}\right)}{\sum_{c^{\prime \prime}} T_{c^{\prime \prime}}^{J^{\pi}\left(r^{\prime} s^{\prime \prime}\right)}\left(E_{c^{\prime \prime}}\right)} \times W_{c f}
$$

where $\sigma_{c}^{J^{\pi}}$ is the compound nucleus formation cross section for a given $(J, \pi), \bar{P}_{f}$ is the average fission probability and $W_{c f}$, the customary in-outgoing channel width fluctuation correction factor.

\footnotetext{
a e-mail: olivier.bouland@cea.fr
} 

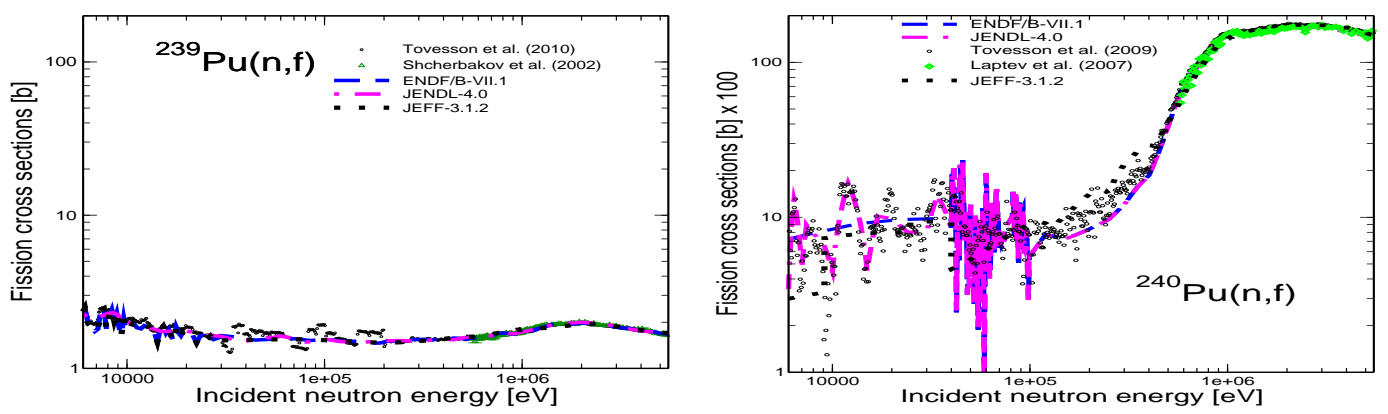

Figure 1. (Color online) Current evaluations (ENDF/B-VII.1, JEFF-3.1.2 and JENDL-4.0) compared to recent measurements by Tovesson et al. [3] and Shcherbakov et al. [4] or by Tovesson et al. [5] and Laptev et al. [6] respectively for the ${ }^{239} \mathrm{Pu}$ and ${ }^{240} \mathrm{Pu}$ target nuclides.

\section{Formal R-matrix and eigenstate classification}

A variable $\beta$, which governs the collective elongation of the system along the fission path, was introduced by Lynn [8]. The Hamiltonian operator is split such that

$$
H=H_{\beta}+H_{\text {int }}\left(\zeta ; \beta_{0}\right)+H_{c}\left(\beta, \zeta ; \beta_{0}\right)
$$

where $H_{\text {int }}$ is the term that governs the excitation of all other degrees of freedom, noted $\zeta$, at a fixed deformation $\beta_{0}$ and $H_{c}$, the coupling Hamiltonian describing the interaction between the $\beta$ mode and the other modes of excitation. The eigenfunctions and eigenvalues of $H_{\text {int }}$ and $H_{\beta}$ are respectively the $\left(\chi_{\mu}\right.$ and $\left.\varepsilon_{\mu}\right)$ and $\left(\phi_{\nu}(\beta)\right.$ and $\left.\varepsilon_{v}\right)$ couples. It is convenient to expand the eigenstates $X_{\lambda}$ of the formal R-matrix states $\lambda$ of the internal region in terms of product pairs of the quasi-vibrational functions $\phi_{\nu}(\beta)$ with the intrinsic functions $\chi_{\mu}$ defined at some special deformations $\beta_{0}$ such that

$$
X_{\lambda}^{\beta_{0}}=\sum_{\nu \mu} C_{\lambda(v \mu)} \phi_{v(\mu)}^{\beta_{0}} \chi_{\mu}
$$

The formal $R$-matrix compound nucleus eigenstates $X_{\lambda}$, which can be qualified asymptotically as either of quasi-class-I (corresponding to ground-state) or quasi-class-II (fission isomer) depending on the location of the largest vibrational amplitude, contain a mixture of the auxiliary class-I and class-II states (with $C_{\lambda(v \mu)}$, the admixture coefficients into the formal states $\lambda$ ). The $X_{\lambda}$ are to be obtained from the diagonalization of $H$ (Eq. 2), which implies the determination of the sub-matrix mixing elements $\left\langle X_{\lambda_{I}}^{(I)}\left|H_{c}\right| X_{\lambda_{I I}}^{(I I)}\right\rangle$ (or the reciprocal elements) characterizing the residual interaction between the $\lambda_{I}$ and $\lambda_{I I}$ compound nucleus state sets. These elements have the following form

$$
\left\langle X_{\lambda_{I}}^{(I)}\left|H_{c}\right| X_{\lambda_{I I}}^{(I I)}\right\rangle \equiv\left\langle\lambda_{I}\left|H_{c}\right| \lambda_{I I}\right\rangle=\sum_{\mu^{\prime \prime} v_{I I}} \sum_{\mu^{\prime} v_{I}}\left\langle\lambda_{I} \mid \mu^{\prime} v_{I}\right\rangle\left\langle\mu^{\prime} v_{I}\left|H_{c}\right| \mu^{\prime \prime} v_{I I}\right\rangle\left\langle\mu^{\prime \prime} v_{I I} \mid \lambda_{I I}\right\rangle
$$

If the intermediate barrier between the two wells is high relatively to the excitation energy, the overlap between the class-I and -II vibrational states is small and the mixing element (Eq. 4) is weak, and the class-II states (with relatively high vibrational amplitudes at the outer barrier) then appear as clear intermediate resonances in the observed fission cross section. 


\subsection{Nature of class-I and class-II compound nucleus states}

By nature the class-I states exhibit very small fission widths, $\Gamma_{\lambda_{I}(f)}$, and are characterized by a small mean spacing (noted $D_{I}$; usually of about a few $\mathrm{eV}$ or a tens of eV respectively for a fissile and a fertile heavy isotope at neutron emission energy). By contrast class-II states exhibit much larger fission widths as well as a larger mean spacing of about $50 \times D_{I}$ at the same excitation energy. The classII neutron width can be neglected since the compound nucleus is originally formed in the groundstate (first well) and because neutron emission from second well is highly contested by fission decay. Thereof, class-I and class-II total widths, noted respectively $\Gamma_{\lambda_{I}(t o t)}$ and $\Gamma_{\lambda_{I I}(t o t)}$, are established as follow

$$
\begin{aligned}
& \Gamma_{\lambda_{I}(t o t)} \approx \Gamma_{\lambda_{I}(n)}+\Gamma_{\lambda_{I}\left(n^{\prime}\right)}+\Gamma_{\lambda_{I}(\gamma)} \\
& \Gamma_{\lambda_{I I}(t o t)} \approx \Gamma_{\lambda_{I(\downarrow)}}+\Gamma_{\lambda_{I I(\uparrow)}}+\Gamma_{\lambda_{I I}(\gamma)}
\end{aligned}
$$

where $\Gamma_{\lambda_{I I}(\uparrow)}$ and $\Gamma_{\lambda_{I I}(\downarrow)}$ are respectively the class-II fission and coupling widths which definition is given in the paragraph below.

\subsection{Formal $R$-matrix concept of class-II partial widths}

On the basis of the classic narrow resonance approximation applied to the transmission coefficient established by Moldauer [9], $\Gamma_{\lambda_{I I}(\downarrow)}$ is obtained by an average over neighboring class-II states and is related to the inner barrier transmission coefficient, $T_{A}$, and similarly, the average class-II fission width is related to the outer fission barrier transmission coefficient, $T_{B}$. It follows

$$
2 \pi\left\langle\Gamma_{\lambda_{I \downarrow}}\right\rangle_{I I} / D_{I I}=T_{A}, \quad 2 \pi\left\langle\Gamma_{\lambda_{I \uparrow}}\right\rangle_{I I} / D_{I I}=T_{B} .
$$

The concept of class-II state coupling width connects a selected class-II state (eventually several), $\lambda_{I I}$, with its neighboring class-I levels, $\lambda_{I_{i}}$, across the inner fission barrier by average of the squared coupling matrix mixing elements, $\left\langle\lambda_{I I}\left|H_{c}\right| \lambda_{I}\right\rangle$, such that

$$
\Gamma_{\lambda_{I \downarrow}}(\alpha)=2 \pi\left\langle\left\langle\lambda_{I I}\left|H_{c}\right| \lambda_{I}\right\rangle^{2}\right\rangle_{I} / D_{I}
$$

\section{Formal R-matrix average fission cross section}

In presence of a double-humped fission barrier, two main cases are generally assumed for calculating either the average fission probability, $\bar{P}_{f}$ or equivalently the effective fission transmission coefficient, $T_{f}$, of Eq.1. At excitation energies well above the fission barrier energy, we can assume that there is a statistical equilibrium among all degrees of freedom in the compound nucleus. Under this hypothesis, the effective fission transmission coefficient across a specified Bohr [10] outer fission barrier channel $\mu$ is

$$
T_{f}^{\mu}=\left(T_{A} T_{B}^{\mu}\right) /\left(T_{A}+T_{B}+T_{I I(\gamma)}+T_{I I(x)}\right),
$$

where $T_{I I(\gamma)}$ and $T_{I I(x)}$ are respectively the (very small) transmission coefficients for decay of classII states by radiative and particle emission. $T_{A}$ and $T_{B}$ are the total fission transmission coefficients over respectively barriers $A$ and $B$ and, are calculated commonly from the well-known Hill-Wheeler expression [11]. At sub-barrier and near-barrier excitation energies, the detailed structure of classII levels has significant impact on $T_{f}$. If the bulk of the strength of $T_{f}$ is concentrated in a narrow energy interval about a class-II level, the average fission probability will vary strongly in the vicinity of this level. In the neighborhood of a single class-II level and on the assumption of uniform class-I 
and class-II level spacings (so-called 'picket fence model'), Lynn and Back [12] have worked out the formulation of the average fission probability $\left(\bar{P}_{f}\right)$ corresponding to barrier tunneling with moderately weak class-I class-II coupling such that

$$
\bar{P}_{f}=\left[1+\left(\frac{T_{I}}{T_{f}}\right)^{2}+\left(\frac{2 T_{I}}{T_{f}}\right) \operatorname{coth}\left(\frac{T_{A}+T_{B}}{2}\right)\right]^{-1 / 2},
$$

with $T_{I}$, being the total class-I transmission coefficient including all open reactions within first well.

\subsection{Coupling and fission width distribution correlations: the $W_{I I}$ factor}

Under both the hypothesis of Eq. 9 and the concept developed in section 3.2, we can express the average over neighboring class-II states of the overall fission transmission coefficient, $T_{f}$, in terms of individual class-II coupling and fission widths such that,

$$
\left\langle T_{f}\right\rangle_{\lambda_{I I}} \approx\left\langle\left(T_{A} T_{B}\right) /\left(T_{A}+T_{B}\right)\right\rangle_{\lambda_{I I}}=\left(2 \pi / D_{I I}\right)\left\langle\left(\Gamma_{\lambda_{I I} \downarrow} \Gamma_{\lambda_{I I} \uparrow}\right) / \Gamma_{\lambda_{I I}}\right\rangle_{\lambda_{I I}}
$$

where, $\Gamma_{\lambda_{I I}}$ represents the total width of a single class-II state. Since we intuitively expect that the statistical fluctuations of the class-II partial fission widths exhibit an independent Porter-Thomas [13] $(v=1)$ distribution across each fully open Bohr fission channel and, the coupling width distribution across the inner Bohr fission channels (considered as a whole - meaning an aggregate of channels) to belong to the chi-squared family (with $v_{e f f}$ the effective value of the number of Degrees of Freedom $(\mathrm{DoF})$ ), we anticipate that correlations between coupling and fission class-II width distributions will be acting similarly to the correlations between the incident-neutron channel and the overall fission decay channel width distributions. Thus we equate

$$
\left\langle\left(\Gamma_{\lambda_{I I} \downarrow} \Gamma_{\lambda_{I I} \uparrow}\right) / \Gamma_{\lambda_{I I}}\right\rangle_{\lambda_{I I}}=\sum_{\mu} W_{I I}(\mu)\left(\left\langle\Gamma_{\lambda_{I I} \downarrow}\right\rangle\left\langle\Gamma_{\lambda_{I I} \uparrow}(\mu)\right\rangle\right) /\left\langle\Gamma_{\lambda_{I I}}\right\rangle,
$$

where $W_{I I}(\mu)$ is introduced as the width fluctuation correction factor, related to a selected fully open Bohr fission channel $(\mu)$, raised by the presence of the class-II states.

Finally, the standard average fission cross section formulation supplied by Eq. 1 must be weighted by the above $W_{I I}$ contribution

$$
\bar{\sigma}_{c f}^{J^{\pi}}\left(E_{n}\right)=\sum_{J} \sigma_{c}^{J^{\pi}}\left(E_{n}\right) \times \bar{P}_{f} \times W_{c f} \times W_{I I}
$$

From Fig. 2 we can verify that both the IS (Lynn and Back formulation of $\bar{P}_{f}$ - Eq. 10 - normalized to the value obtained assuming statistical regime - Eq. 9) and overall width fluctuation correction factor $\left(W_{n, f} * W_{I I}\right.$ product) exert a strong influence on the average fission cross section magnitude. Depending on fission barrier heights relatively to the neutron threshold energy, the IS effect is either much smaller than the overall width statistical fluctuation correction as for the ${ }^{239} \mathrm{Pu}$ fissile target nucleus (left-side Figure) or much larger for a non-fissile target nucleus as for ${ }^{240} \mathrm{Pu}$ (right-side Figure).

\section{Nested Monte carlo fission cross section calculations}

A powerful alternative to analytical expressions valid under specific conditions, such as the statistical regime or the Lynn and Back [12] average fission sub-barrier probability $\left(\bar{P}_{f}\right)$ described above, is 

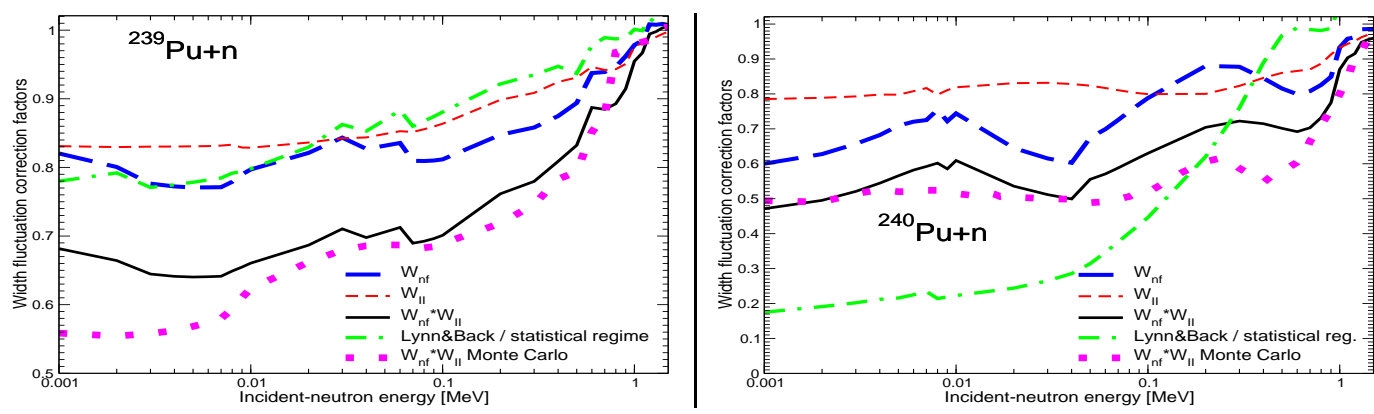

Figure 2. (Color online) $W_{n, f}$ and $W_{I I}$ width fluctuation correction factors (and product - black-solid line) calculated as function of neutron energy respectively for the ${ }^{239} \mathrm{Pu}$ and ${ }^{240} \mathrm{Pu}$ target nuclides. For comparison, the reduction of the average fission barrier probability due to barrier tunneling (estimated from the Lynn and Back formula - Eq. 10 - normalized to the statistical regime - Eq. 9) is plotted (red-short-dashes) as well as the fluctuation factor product extracted from the accurate Monte Carlo procedure (purple-dotted line).

the Monte Carlo-type (MC) method. Our MC procedure [2] presents the advantage of providing average cross sections taking full account of all statistical nuclear data parameter fluctuations under in-situ IS coupling conditions. Our present approach simulates the $R$-matrix resonance properties of a selected class-II state and those of the overlapped neighboring class-I states over at least a full class-II energy spacing, using a chain of pseudo-random numbers for a fine-tuned selection process based on both level width and spacing statistical distributions with suitable averages and in particular the Porter-Thomas width distributions of the dedicated elements $\left(\left\langle\lambda_{I}\left|H_{c}\right| \lambda_{I I}\right\rangle^{2}, \Gamma_{\lambda_{I \downarrow} \downarrow}\right.$ and $\Gamma_{\lambda_{I \uparrow \uparrow}}$ as introduced by Eqs. 8 and 7), were simulated to model the full extent of IS fluctuations. The extraction of the $W_{n, f} * W_{I I}$ undecoupled product from the more accurate MC procedure can be compared to the analytical estimate obtained previously (Fig. 2). We observe that the effect of the $W_{n, f} * W_{I I} \mathrm{MC}$ product is still larger than the one given by the analytical estimate. The effect on the simulated average cross section will be to lower it still below the magnitude it would have using the analytical estimate.

\subsection{Overall fission barrier effective degree of freedom}

A collateral damage of the IS, usually poorly addressed, is the reduction of the effective degree of freedom, $v_{f_{e f f}}$, of the overall fission barrier. The precise value is crucial because this latter has to be input into the calculation of the standard $W_{n, f}$ factor. For standard Hauser-Feshbach statistical theory with adequate width fluctuation correction factor, $v_{f_{e f f}}$ should be equal to the number of open channels at the outer barrier. However the plausible restriction of the fission transmission across the outer fission channels, due to the IS, will reduce $v_{f_{e f f}}$ significantly. Hopefully, the correct value of $v_{f_{e f f}}$ can be estimated from thousand MC calculations with the assumption that, at near-barrier energy, the class-II total width is large and that its strength is meanly spread through its coupling width over many class-I states $\left(\Gamma_{\lambda_{I I} \downarrow}>>\Gamma_{\lambda_{I I} \uparrow}\right)$. The mixing elements are then somewhat large (so-called moderately weak coupling) and the class-II state fission width is mixed in a Lorentzian pattern into the formal $R$-matrix states. From those MC calculations, the effective degree of freedom of the overall barrier fission width can be retrieved from means and variances. An example of reduction of $v_{f_{e f f}}$ is given in Table 1 respectively for the ${ }^{239} \mathrm{Pu}$ and ${ }^{240} \mathrm{Pu}$ target nuclides and at two incident-neutron energies 
representative of the URR. We can guess that the resulting standard $W_{n, f}$ factor will be significantly altered.

\begin{tabular}{|c||c|c|}
\hline$v_{\text {eff }}$ & ${ }^{240} \mathrm{Pu}^{*}\left(J^{\pi}=0^{+}\right)$ & ${ }^{241} \mathrm{Pu}^{*}\left(J^{\pi}=0.5^{+}\right)$ \\
\hline \hline $1 \mathrm{keV}$ & 0.72 & 0.66 \\
\hline $100 \mathrm{keV}$ & 0.74 & 0.66 \\
\hline
\end{tabular}

Table 1. IS impact in terms of degree of freedom respectively for the ${ }^{239} \mathrm{Pu}$ and ${ }^{240} \mathrm{Pu}$ target nuclides normalized to a single fully open outer barrier channel. Without IS (such that the single hump picture), the distribution of the formal R-matrix eigenstate fission widths is expected to follow a Porter-thomas distribution with $v_{f}=1$.

\section{Conclusions}

The purpose here was to recall and emphasize the existence of the class-II width fluctuation correction factor which lowers the average fission cross section of about $20 \%$ for a fertile isotope over the URR. This paper also demonstrates that its effect is also non negligible $(>10 \%)$ for a fissile isotope. Another goal was to enlighten a collateral damage of the IS, usually poorly addressed, which is the drastic reduction of the degree of freedom of the overall fission barrier to be input in the calculation of the standard $W_{n, f}$ factor. Its value will be strongly modified. Although standard evaluation works can by-pass the latter corrections by playing on other parameter types (e.g; fundamental fission barrier heights), those short-cuts will be an additional source of uncertainties on predictions to be made for nuclides which cross sections can not be measured.

\section{Acknowledgment}

This work is made in collaboration with Dr. Patrick Talou and Dr. J. Eric Lynn from the T-2 group of LANL. O. Bouland expresses his deep gratitude for enlightening discussions with those fellows and to Dr. Olivier Serot from CEA Cadarache for paper review.

\section{References}

[1] F. H. Froehner, Nucl. Sci. Eng. 111, 404 (1992).

[2] O. Bouland, J. E. Lynn and P. Talou, EPJ Web of Conf. DOI: 10.1051, 2108004 (2012).

[3] F. Tovesson and T. S. Hill, Nucl. Sci. Eng. 165, 224 (2010).

[4] O. A. Shcherbakov et al., Proc. of ASAP 2002, Ed. P. E. Koehler, 123 (2002).

[5] F. Tovesson et al., Phys. Rev. C 79, 014613 (2009).

[6] A. Laptev et al., Proc. of $3^{\text {rd }}$ International Conf. on Fis. and Properties of Neutron-Rich Nuclei, Sanibel Island, FL (2002).

[7] W. Hauser and H. Feshbach, Phys. Rev. 87, 366 (1952).

[8] J. E. Lynn, J. Phys. A: Math. Nucl. Gen., 6, 542 (1973).

[9] P. A. Moldauer, Phys. Rev. 123, 3, 968 (1961).

[10] A. Bohr, Peaceful Uses of Atomic Energy, 2, 220, United Nations (1955).

[11] D. L. Hill and J. A. Wheeler, Phys. Rev. 89, 1102 (1953).

[12] J. E. Lynn and B. B. Back, J. Phys. A: Math. Nucl. Gen., 7, No. 3, 395 (1974).

[13] C. F. Porter and R. Thomas, Phys. Rev. 104, 483 (1956). 